\title{
GAMIFICATION IN HIGHER EDUCATION AND STEM: A SYSTEMATIC REVIEW OF LITERATURE
}

\author{
Margarita Ortiz ${ }^{1}$, Katherine Chiluiza ${ }^{2}$, Martin Valcke ${ }^{3}$ \\ ${ }^{1}$ Ghent University (BELGIUM) / Information Technology Center CTI-ESPOL (ECUADOR) \\ ${ }^{2}$ Escuela Superior Politécnica del Litoral - ESPOL (ECUADOR) \\ ${ }^{3}$ Ghent University (BELGIUM)
}

\begin{abstract}
In recent years, gamification, the use of game elements in non-game contexts, has drawn the attention of educators due to the possibility of making learning more motivating and engaging; this led to an increase of research in the field. Despite the availability of literature reviews about gamification and its effects, no work to this date has focused exclusively on Higher Education (HE). Next, worldwide there is an increasing demand for skilled Science, Technology, Engineering and Mathematics (STEM) professionals that meet the challenges related to scientific and technological innovations of the 21st Century. This lead to the need of strengthening STEM Higher Education. This brings us to the purpose of this work: presenting a systematic literature review of empirical studies about gamification STEM related Higher Education. This review study started from a systematic mapping design of 'Web of Science' articles, with following inclusion criteria: empirical gamification studies set up in HE, published between 2000 and 2016; focusing on undergraduate or graduate students; in the STEM knowledge field, and set up in authentic settings. An initial search resulted in 562 potentially relevant articles. After applying all selection criteria, only 18 studies could be retained. 12 additional articles were included by analyzing references from earlier literature reviews, resulting in 30 studies to be included. Analysis results show how a combination of game elements (e.g. leaderboards, badges, points and other combinations) positively affects students' performance, attendance, goal orientation and attitude towards mostly computer science related subjects. The analysis results also point at a lack of studies in certain STEM areas, a lack of studies that identify the particular game element associated with the positive differential impact on student performance; a lack of validated psychometric measurements, and lack of focus on student variables that could/should be taken into account as mediating/moderating variables clarifying the impact of gamification in the $\mathrm{HE}$ focus on STEM learning and teaching.
\end{abstract}

Keywords: Gamification, STEM, review of research, literature review.

\section{INTRODUCTION}

Since 2010, when gamification became widespread [1], it has been used in different settings such as commerce [2], health [3], and work [4] but above all, in education [5], [6]. The vast amount of studies in this field, when revising literature reviews, shows how important gamification has become for educators in terms of evaluating its effect on students' learning [6] [7]. Another important topic of educators' interest is STEM (Science, Technology, Engineering and Mathematics) education. The Obama Administration in the United States [8] as well as The Horizon 2020, representing Europe [9], emphasize the importance of preparing young people for society related scientific and technological innovations, and assure that a proper STEM preparation equips graduates excelling in those fields. In this context, using gamification in STEM related areas could be one way to engage and motivate students to pursue undergraduate and graduate careers. Hence, the importance of analyzing and understanding how gamification has been used in Higher Education within a STEM context, to use its methodological approaches in current educational programs.

In this study, we present a systematic review of academic literature on gamification in Higher Education within a STEM context. Our goals are (a) to provide a current state-of-the-art of empirical work regarding gamification and (b) to find gaps in existing studies. The latter helps drafting directions for future research. This work is organized as follows: First, related work about gamification is presented, followed by a detailed methodology on how this review was carried out. Next, results and discussion sections are described. Finally, limitations as well as conclusions with suggested ideas for further research are presented. 


\section{RELATED WORK}

To the best of our knowledge, there are not reviews of research that tackle exclusively the use of gamification in Higher Education within STEM fields. Nevertheless, we can find studies that provide the state of the art in the field of gamification from a broad to a more specific perspective. For instance, in [10], empirical studies focus on the motivational affordances and, psychological and behavioral outcomes of gamification. Researchers concluded that although the results are generally positive, there are underlying confounding factors that should be taken into consideration such as the context and quality of users. Another study [11], adopted a theoretical and empirical point of view, due to underdeveloped theoretical foundations of gamification, conceptual ambiguity and contradictory uses. These authors stressed a conceptualized definition of the term, outlined critical game elements, and compared gamification to other approaches such as games with purpose or alternative reality games. Findings showed there are three areas raising concern: subjectivity in definitions, incongruities among empirical studies, and inadequate experimental designs. Some reviews, focused exclusively on particular educational contexts [12], [7], [6]. In [12], conclusions pointed out that studies mistakenly use the term gamification and how the concept is used as a synonym for Game-based learning, something also found in [11]. More recently, more clear definitions are being presented, that additionally stress links between gamification and motivation, engagement and learning outcomes [11]. These authors stress more experimental work is needed to study this complex interplay. Furthermore, in [6], a new playfield is added, when studies are examined based on game design principles, types of application used (e.g. MOOCs, blended learning, etc.), work distribution among players/subjects, etc. They highlight the importance of technical support and a strong need for experimental evaluative work. The latter is critical to be able to evaluate the particular impact of individual gamification elements. Recently, more studies are being published, focusing on a more particular knowledge domain; for instance, games and gamification in engineering students [13] or gamification in software engineering [14]. Though these studies point at the impact on learning performance and interaction effects with motivation, the authors reiterate consistently the need for more controlled empirical research to truly measure the impact of gamification.

\section{METHOD}

To conduct this review, the five stage framework of Arkey and O' Malley [15] was used to follow a rigorous approach, enabling replication of the search strategy and assuring reliability of study findings. The framework consists of five stages, explained below.

\subsection{Stage 1: Identifying the research question}

We decided to guide our search, posing five questions, based on questions explored in [6], [10], [11].

- $Q 1=$ What gamification elements have been used in Higher Education within STEM fields?

- Q2= Which STEM fields have experimented with gamification?

- $\mathrm{Q} 3=$ What have been the results of the studies?

- Q4 = What variables and data collection methods have been used?

- Q4= How much time are students involved in the gamification experience?

\subsection{Stage 2: Identifying relevant studies}

The selection process started in March 3, 2016 and lasted about one month. We used the academic search service "Web of Science". As in previous studies [6], [10], [11], [12], [14], to guarantee a broad coverage, we also used the keywords "gamification" and "gamif" and search title and content fields. As a time frame we put forward the period from 2000-2016. In terms of language, due to one of the author's bilingualism, Spanish and English studies were considered. In addition, only peer reviewed studies were included in the search.

\subsection{Stage 3: Study Selection}

The selection process resulted in a dataset of 562 articles. From reading the abstracts, we realized there was a large amount of irrelevant articles. They focused for instance on other educational areas, e.g. elementary and secondary, or used the word gamification as a synonym of other game-related topics such as serious games, video games, etc. Considering this result, we defined detailed inclusion 
and exclusion criteria as reflected in Table 1. Once applied to the original dataset, only 18 articles remained. To enrich this basic set, we started an additional selection phase, this time building on the reference lists of related reviews of research. This resulted in 12 additional articles, in line with the inclusions/exclusion criteria put forward and resulting in 30 studies to be analyzed. Figure 1, illustrates the process of article selection from stage 2 and 3.

Table 1: In Depth Inclusion and Exclusion criteria.

\begin{tabular}{|c|c|c|c|}
\hline Criterion & Inclusion & Exclusion \\
\hline Topic & $\begin{array}{c}\text { Gamification as } \\
\text { defined by [1] }\end{array}$ & Using gamification to refer to game based learning, serious games, \\
games, video games
\end{tabular}

Studies excluded by:

Topic $(n=196)$

Educational Level $(n=264)$

Participants $(n=1)$

Study Focus $(n=42)$

Knowledge area $(n=28)$

Other $=(n=13)$

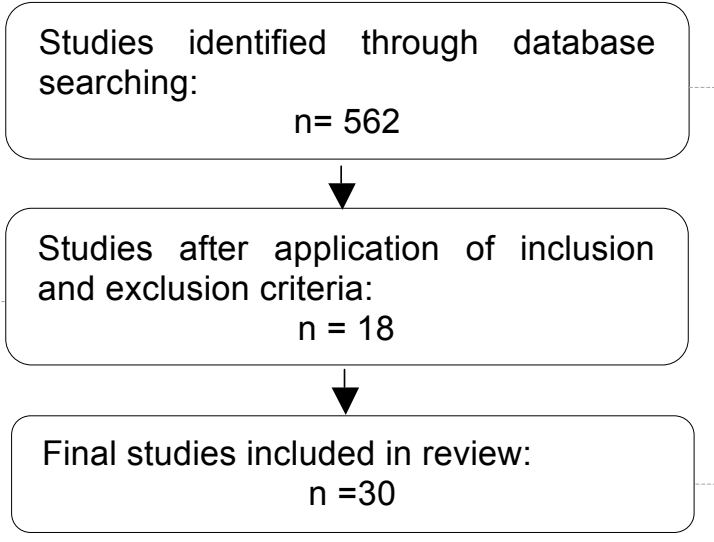

Studies identified through database $n=562$ Time, Language, peer reviewed)

\begin{tabular}{l} 
Studies included \\
based on new \\
search $\quad$ of \\
references in \\
reviews of literature \\
\multicolumn{2}{c}{$(n=12)$}
\end{tabular}

Fig 1. Article Selection Process (Stages 2 and 3).

\subsection{Stage 4: Charting the Data}

In this stage, the selected studies are organized in a way helping readers understand the data. Summaries were developed for each articled based on the author's last names, country and year of publication, method, sample size, gamification element, course subject, variables, duration and outcome. A detailed view of studies is provided in Table 2.

\subsection{Stage 5: Collating, summarizing and reporting the results}

This final stage summarizes and reports the results. This stage is explained in depth in the next section.

\section{RESULTS}

This section describes the distribution of published work on each classification criterion provided in Table 2 . 
Table 2. Selected Studies.

\begin{tabular}{|c|c|c|c|c|c|c|c|c|}
\hline Study & $\begin{array}{l}\text { Country } \\
\text { and Year }\end{array}$ & Method & $\mathbf{N}$ & $\begin{array}{l}\text { Gamification } \\
\text { Element }\end{array}$ & Course Subject & Variables & Duration & Outcome \\
\hline [19] & $\begin{array}{l}\text { Brazil } \\
(2016)\end{array}$ & Mixed & 36 & $\begin{array}{l}\text { Badges } \\
\text { Challenges }\end{array}$ & $\begin{array}{l}\text { Science and } \\
\text { Technology, } \\
\text { Biotechnology }\end{array}$ & engagement & 3 weeks & Positive \\
\hline [20] & $\begin{array}{l}\text { Colombia } \\
(2015)\end{array}$ & Quantitative & 2263 & $\begin{array}{c}\text { Badges, Points, } \\
\text { Leaderboard, } \\
\text { Levels }\end{array}$ & Pre-calculus & $\begin{array}{c}\text { academic } \\
\text { achievement }\end{array}$ & 17 weeks & Positive \\
\hline [21] & $\begin{array}{l}\text { Israel } \\
(2015)\end{array}$ & Quantitative & 38 & $\begin{array}{c}\text { Points, Badges, } \\
\text { rewards } \\
\text { Leaderboard, } \\
\text { progress bar }\end{array}$ & $\begin{array}{c}\text { Software } \\
\text { Analysis and } \\
\text { Design }\end{array}$ & playfulness & $\begin{array}{c}1 \\
\text { semester }\end{array}$ & Mixed \\
\hline [22] & $\begin{array}{l}\text { South K. } \\
(2015)\end{array}$ & Quantitative & 33 & $\begin{array}{l}\text { Leaderboard, } \\
\text { virtual money, } \\
\text { characters, } \\
\text { badges } \\
\end{array}$ & $\begin{array}{l}\text { Not specified } \\
\text { (Engineering } \\
\text { class) }\end{array}$ & motivation & $\begin{array}{c}1 \\
\text { semester }\end{array}$ & Mixed \\
\hline [23] & $\begin{array}{l}\text { Finland } \\
(2015)\end{array}$ & Quantitative & 469 & Badges & $\begin{array}{l}\text { Data Structures } \\
\text { and Algorithms }\end{array}$ & $\begin{array}{c}\text { Study practices, } \\
\text { achievement goal } \\
\text { orientation }\end{array}$ & $\begin{array}{c}1 \\
\text { semester }\end{array}$ & Mixed \\
\hline [24] & $\begin{array}{l}\text { Finland } \\
(2015)\end{array}$ & Quantitative & 281 & Badges & $\begin{array}{l}\text { Data Structures } \\
\text { and Algorithms }\end{array}$ & $\begin{array}{c}\text { Behavior and } \\
\text { attitude towards } \\
\text { badges }\end{array}$ & $\begin{array}{c}1 \\
\text { semester }\end{array}$ & Mixed \\
\hline [25] & $\begin{array}{l}\text { Finland } \\
(2014)\end{array}$ & Quantitative & 278 & Badges & $\begin{array}{l}\text { Data Structures } \\
\text { and Algorithms }\end{array}$ & $\begin{array}{c}\text { Behavior, } \\
\text { achievement goal } \\
\text { orientation }\end{array}$ & 4 weeks & Neutral \\
\hline [26] & $\begin{array}{l}\text { Finland } \\
(2013)\end{array}$ & Quantitative & 281 & Badges & $\begin{array}{l}\text { Data Structures } \\
\text { and Algorithms }\end{array}$ & behavior & $\begin{array}{c}1 \\
\text { semester }\end{array}$ & Mixed \\
\hline [27] & $\begin{array}{l}\text { Finland } \\
(2014)\end{array}$ & Quantitative & 162 & Badges & $\begin{array}{l}\text { Data Structures } \\
\text { and Algorithms }\end{array}$ & behavior & $\begin{array}{c}1 \\
\text { semester }\end{array}$ & Neutral \\
\hline [28] & $\begin{array}{l}\text { Spain } \\
(2015)\end{array}$ & Quantitative & 86 & $\begin{array}{c}\text { Badges } \\
\text { Leaderboards }\end{array}$ & $\begin{array}{l}\text { Computer } \\
\text { Networks }\end{array}$ & $\begin{array}{c}\text { academic } \\
\text { performance }\end{array}$ & 14 weeks & Positive \\
\hline [29] & $\begin{array}{l}\text { Spain } \\
(2014)\end{array}$ & Quantitative & 22 & $\begin{array}{c}\text { Badges } \\
\text { leaderboards }\end{array}$ & $\begin{array}{l}\text { C programming } \\
\text { language }\end{array}$ & $\begin{array}{c}\text { engagement and } \\
\text { academic } \\
\text { performance }\end{array}$ & 1 week & Positive \\
\hline [30] & $\begin{array}{l}\text { Sweden } \\
(2015) \\
\end{array}$ & Mixed & 271 & $\begin{array}{c}\text { Progress bars } \\
\text { Badges }\end{array}$ & $\begin{array}{l}\text { Game Based } \\
\text { Learning }\end{array}$ & motivation & $\begin{array}{c}1 \\
\text { semester } \\
\end{array}$ & Mixed \\
\hline [31] & $\begin{array}{l}\text { Estonia } \\
(2014)\end{array}$ & Quantitative & 32 & $\begin{array}{l}\mathrm{XP} \text {, avatar, big } \\
\text { boss, score, } \\
\text { chance, } \\
\text { competition }\end{array}$ & $\begin{array}{c}\text { Game Interaction } \\
\text { Computer } \\
\text { Games }\end{array}$ & $\begin{array}{l}\text { motivation } \\
\text { flow }\end{array}$ & $\begin{array}{c}1 \\
\text { semester }\end{array}$ & Positive \\
\hline [32] & $\begin{array}{l}\text { Estonia } \\
(2014)\end{array}$ & Quantitative & 76 & $\begin{array}{c}\text { Avatar, Points, } \\
\text { leaderboard, } \\
\text { levels }\end{array}$ & $\begin{array}{c}\text { Research } \\
\text { Methods to ICT } \\
\text { students } \\
\end{array}$ & immersion & $\begin{array}{c}1 \\
\text { semester }\end{array}$ & Mixed \\
\hline [33] & $\begin{array}{l}\text { Denmark } \\
(2014)\end{array}$ & Qualitative & 20 & points & $\begin{array}{c}\text { Gamification: } \\
\text { Digital Games in } \\
\text { everyday spaces }\end{array}$ & $\begin{array}{l}\text { motivation } \\
\text { flow }\end{array}$ & $\begin{array}{l}24 \text { hours } \\
\text { In } 6 \\
\text { sessions }\end{array}$ & Mixed \\
\hline [34] & $\begin{array}{l}\text { Not stated } \\
(2014)\end{array}$ & Mixed & 70 & Quest & Computing & $\begin{array}{c}\text { students' } \\
\text { engagement and } \\
\text { achievement }\end{array}$ & $\begin{array}{c}1 \\
\text { semester }\end{array}$ & Positive \\
\hline [35] & $\begin{array}{l}\text { Austria } \\
(2014) \\
\end{array}$ & Quantitative & 50 & Challenge & $\begin{array}{c}\text { Software } \\
\text { Development }\end{array}$ & engagement & 14 weeks & Positive \\
\hline [36] & $\begin{array}{l}\text { Austria } \\
(2014)\end{array}$ & Mixed & 27 & $\begin{array}{l}\text { Badges, } \\
\text { Leaderboard }\end{array}$ & $\begin{array}{c}\text { Information } \\
\text { Search and } \\
\text { Retrieval (ISR) } \\
\end{array}$ & $\begin{array}{l}\text { engagement and } \\
\text { motivation }\end{array}$ & $\begin{array}{c}1 \\
\text { semester }\end{array}$ & Positive \\
\hline [37] & $\begin{array}{c}\text { UK } \\
(2013)\end{array}$ & Quantitative & 136 & Badges & $\begin{array}{c}\text { Game } \\
\text { Production }\end{array}$ & $\begin{array}{c}\text { attendance and } \\
\text { overall grade }\end{array}$ & 1 module & Positive \\
\hline
\end{tabular}




\begin{tabular}{|c|c|c|c|c|c|c|c|c|}
\hline [38] & $\begin{array}{l}\text { Portugal } \\
\text { (2013) }\end{array}$ & Quantitative & 77 & $\begin{array}{l}\text { Points, levels, } \\
\text { badges } \\
\text { leaderboard, } \\
\text { challenges }\end{array}$ & $\begin{array}{l}\text { Multimedia } \\
\text { Content } \\
\text { Production }\end{array}$ & $\begin{array}{l}\text { engagement, } \\
\text { satisfaction and } \\
\text { academic } \\
\text { performance }\end{array}$ & $\begin{array}{c}1 \\
\text { semester }\end{array}$ & Mixed \\
\hline [39] & $\begin{array}{l}\text { Portugal } \\
\text { (2013) }\end{array}$ & Quantitative & 242 & $\begin{array}{c}\text { Points progress } \\
\text { levels } \\
\text { leaderboard } \\
\text { challenges, } \\
\text { badges } \\
\end{array}$ & $\begin{array}{l}\text { Multimedia } \\
\text { Content } \\
\text { Production }\end{array}$ & engagement & 5 years & Positive \\
\hline [40] & $\begin{array}{l}\text { USA } \\
(2013)\end{array}$ & Quantitative & 51 & $\begin{array}{c}\text { XP, levels, } \\
\text { leaderboard, } \\
\text { badges }\end{array}$ & $\begin{array}{l}\text { Chemical } \\
\text { Engineering }\end{array}$ & $\begin{array}{c}\text { engagement, } \\
\text { interest, academic } \\
\text { performance }\end{array}$ & $\begin{array}{c}1 \\
\text { semester }\end{array}$ & Mixed \\
\hline [41] & $\begin{array}{l}\text { USA } \\
(2011)\end{array}$ & Quantitative & n. s. & $\begin{array}{l}\text { Leaderboard, } \\
\text { Ranks, Levels, } \\
\text { virtual currency } \\
\text { XP, time } \\
\text { pressure } \\
\end{array}$ & $\begin{array}{l}\text { Software } \\
\text { Engineering } \\
\text { Technology } \\
\text { Capstone }\end{array}$ & Interest & 3 terms & Mixed \\
\hline$[42]$ & $\begin{array}{c}\text { Canada } \\
(2012)\end{array}$ & Quantitative & 30 & Badges & $\begin{array}{l}\text { Communication } \\
\text { and Design }\end{array}$ & $\begin{array}{c}\text { focus, } \\
\text { engagement, task } \\
\text { performance }\end{array}$ & $\begin{array}{c}\text { Not } \\
\text { Specified }\end{array}$ & Positive \\
\hline [43] & $\begin{array}{l}\text { Germany } \\
(2013)\end{array}$ & Quantitative & 59 & $\begin{array}{c}\text { Points, } \\
\text { challenges, } \\
\text { leaderboard, } \\
\text { levels }\end{array}$ & $\begin{array}{c}\text { Software } \\
\text { Engineering }\end{array}$ & engagement & $\begin{array}{l}1 \text { quarter } \\
\text { semester }\end{array}$ & Negative \\
\hline [44] & $\begin{array}{l}\text { Germany } \\
(2012)\end{array}$ & Quantitative & 10 & Leaderboard & $\begin{array}{c}\text { Extreme } \\
\text { Programming lab } \\
\text { and seminar }\end{array}$ & Task performance & 4 weeks & Mixed \\
\hline [45] & $\begin{array}{l}\text { Germany } \\
(2012)\end{array}$ & Qualitative & 37 & Leaderboard & software project & Behavior & 4 months & Mixed \\
\hline$[46]$ & $\begin{array}{c}\text { Singapore } \\
(2011)\end{array}$ & Quantitative & 51 & $\begin{array}{l}\text { Points, Levels } \\
\text { leaderboard }\end{array}$ & $\begin{array}{l}\text { Computer } \\
\text { Science }\end{array}$ & engagement & $\begin{array}{c}1 \\
\text { semester }\end{array}$ & Positive \\
\hline [47] & $\begin{array}{c}\text { Netherlands } \\
(2014)\end{array}$ & Quantitative & 450 & $\begin{array}{l}\text { Points, levels } \\
\text { leaderboard, } \\
\text { unlocking, } \\
\text { badges } \\
\end{array}$ & $\begin{array}{l}\text { Cloud } \\
\text { Computing, } \\
\text { Computer } \\
\text { Organization }\end{array}$ & engagement & $\begin{array}{c}1 \\
\text { semester }\end{array}$ & Positive \\
\hline [48] & $\begin{array}{c}\text { South Africa } \\
(2013)\end{array}$ & Quantitative & 90 & $\begin{array}{l}\text { Leaderboard, } \\
\text { Hints Points, } \\
\text { Ranks, progress } \\
\text { bar, badges }\end{array}$ & $\begin{array}{c}\text { 2D games } \\
\text { design and } \\
\text { development }\end{array}$ & $\begin{array}{c}\text { lecture } \\
\text { attendance, } \\
\text { content } \\
\text { understanding, } \\
\text { problem solving } \\
\text { skills and } \\
\text { engagement }\end{array}$ & $\begin{array}{c}1 \\
\text { semester }\end{array}$ & Positive \\
\hline
\end{tabular}

\subsection{General Information}

Results show studies related to gamification began from 2011 to the present time. When studies were clustered according to the continent where they belong, to have a broader view, results show that most studies are from Europe (20), followed by America (5), Asia (3), Africa (1) and (1) not specified. This might reflect the impact of the Horizon report mentioning Gamification in 2013 and 2014 as a trend in Higher Education [16] [17]. A decrease of European studies in 2015 might also reflect gamification was not mentioned in last year's report [18]. Dominant European countries are Finland, Austria, Spain, Sweden, Estonia, Denmark, United Kingdom, Portugal, Germany and the Netherlands. Finland reflects most publications (5). Nevertheless, this outcome could be due to studies from the same group of authors. Regarding research methods, the majority of studies apply a quantitative approach. Out of 30 studies, 24 are quantitative in nature, 4 adopt a mixed and only 2 adopt a qualitative approach. In terms of sample size, 13 studies mainly involved 11 to 60 students, followed by 5 studies involving 61 to 110 . The remaining 9 , ranged from 111 to 470 . Only 1 study was limited to a sample size of 10 students or less, only 1 involved a large number (2263 students), and 1 did not specified its sample size. 


\subsection{Research Questions}

\subsubsection{Research question 1: What gamification elements have been used in Higher Education within STEM fields?}

Table 3 shows the different type of gamification elements used in the studies. As a typology, we based our analysis on the alternative gamification elements presented in [11]. Most studies used a combination of gamification elements. These are usually points, badges and leaderboards, plus other elements such as challenges, levels, avatar, etc. Moreover, it is noteworthy to see, that there are only five elements being studied in isolation, mostly badges. This should not be surprising, due to the available research provoked by this element [49]. In contrast, points, challenges, quests and leaderboards are rarely studied as unique gamification elements.

Table 3. Gamification elements.

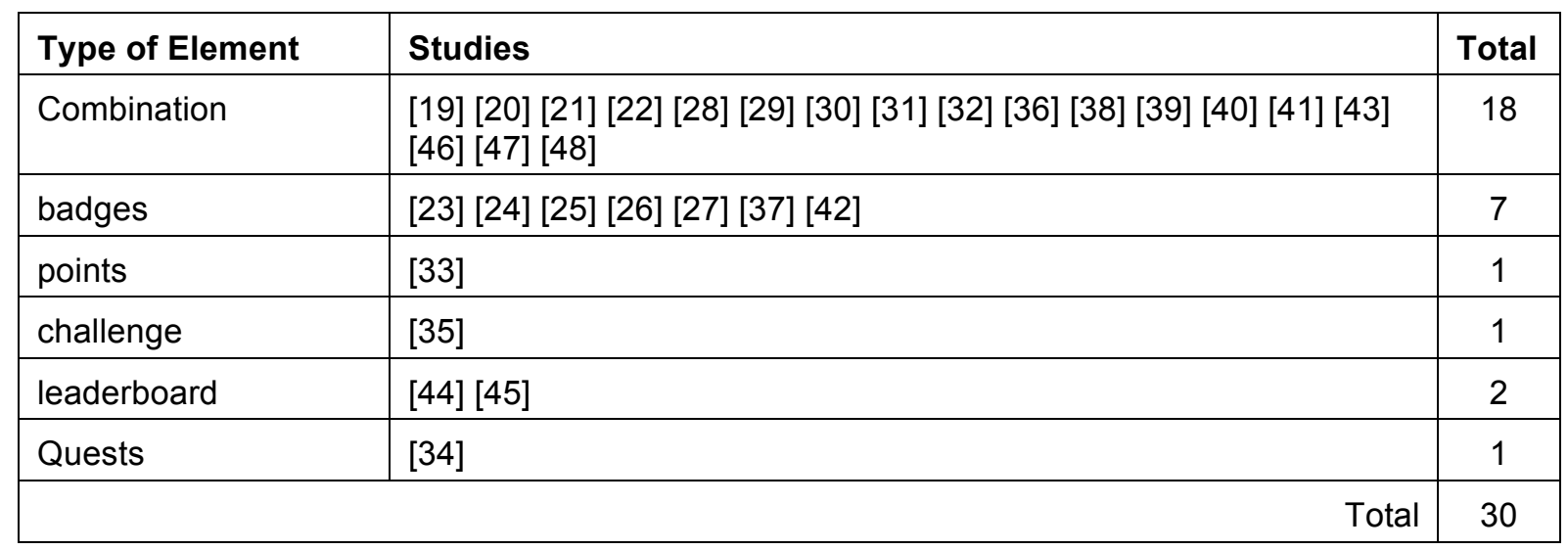

\subsubsection{Research question 2: Which STEM fields have experimented with gamification?}

Table 4 shows how computer science courses dominate the STEM field in which gamification studies take place. There is a minor presence of areas such as Math, Chemistry and Science. These findings are in line with those reported by [6] who also observed a tendency to apply gamification in this particular area.

Table 4. STEM fields experimenting with gamification.

\begin{tabular}{|c|c|c|}
\hline STEM field & Studies & Total \\
\hline Computer Science & $\begin{array}{l}{[21][23][24][25][26][27][28][29][30][31][33][34][35][36][37]} \\
{[38][39][41][42][43][44][45][46][47][48]}\end{array}$ & 25 \\
\hline Science/Technology & [19] [32] & 2 \\
\hline Math & {$[20]$} & 1 \\
\hline Chemistry & {$[40]$} & 1 \\
\hline Not specified & {$[22]$} & 1 \\
\hline \multicolumn{2}{|r|}{ Total } & 30 \\
\hline
\end{tabular}

\subsubsection{Research question 3: What have been the results of the studies?}

Table 5 shows the nature of the research findings. We classified a study as neutral when the conclusions reflected neither an improvement nor a decrease in the dependent variables. Studies could further be classified as positive, negative and mixed. Overall, researchers report a combination of positive and mixed results 
Table 5. Study results.

\begin{tabular}{|l|l|c|}
\hline Result & Studies & Total \\
\hline Positive & {$[19][20][28][29][31][34][35][36][37][39][42][46][47][48]$} & 14 \\
\hline Negative & {$[43]$} & 1 \\
\hline Neutral & {$[25][27]$} & 2 \\
\hline Mixed & {$[21][22][23][24][26][30][32][33][38][40][41][44][45]$} & 13 \\
\hline Total & 30 \\
\hline
\end{tabular}

\subsubsection{Research question 4: What variables and data collection methods have been used?}

Overall, table 2 shows most studies focused on measuring engagement as a way to grasp student motivation towards the new gamified system/class. At a more detailed level, we see that when Learning Management Systems are used, log analysis is part of the methods used to measure engagement. Furthermore, studies measure this same variable based on perceptions via surveys, collecting feedback based on student experiences. To collect qualitative data, interviews and openquestion questionnaires are usually applied. Only in few cases [19], [21] [24], [25], [31], [32] validated psychometric measurements were used to assess personality, flow, motivation and goal orientation.

\subsubsection{Research question 5: How much time students are involved in a gamified experience?}

Table 6 shows the amount of time spent in the gamified experiment. As can be seen, most studies last one semester. In contrast to the study in [10], who criticized studies being short, our review points at an increase in longer term studies.

Table 6. Experiment Timeframes.

\begin{tabular}{|l|l|c|}
\hline $\begin{array}{l}\text { Range of Time } \\
\text { Spent }\end{array}$ & Studies & Total \\
\hline $1-24$ hours & {$[33]$} & 1 \\
\hline $1-14$ weeks & {$[19][20][25][28][29][35][44]$} & 7 \\
\hline $1-4$ months & {$[43][45]$} & 2 \\
\hline 1 semester & {$[21][22][23][24][26][27][30][31][32][34][36][38][40][46][47][48]$} & 16 \\
\hline$\geq 1$ year & {$[39]$} & 1 \\
\hline Not specified & {$[42]$} & 1 \\
\hline Other & {$[37][41]$} & 2 \\
\hline Total & & 30 \\
\hline
\end{tabular}

\section{DISCUSSION}

This review sheds light on empirical studies using gamification within a Higher Education context and in STEM fields, published between 2000 to March 2016. Overall, we observe an increase in interest in the field, especially from European countries.

In terms of research question 1, the fact most studies use multiple game elements, does not allow to know exactly which element is associated with particular effects in students. This conclusion is also shared in the review of [6], hence the importance of setting up research that helps isolating the impact of individual gamification elements. Regarding research question 2, it is clear Computer Science dominates the research field. The lack of necessary skills, from the side of instructors, for creating, adapting, and/or maintaining a technological infrastructure like a LMS, could be linked to this finding, as underlined by [6]. Thus, more technological support is needed to involve other STEM areas in empirical studies. About research question 3 , it is hard to affirm that gamification is associated completely with positive findings. Researchers need to understand the articular nature of each individual study to conclude whether a gamified intervention was beneficial or not for students. 
Compared to an earlier review of the literature [10] hardly reporting positive findings, we observe studies with an improved research design reporting more positive and mixed results. Results are often mixed because many variables are not being taken into consideration when designing the studies; such as motivation, player types, or personality. These should be included in the future research designs to develop a more comprehensive understanding of gamification in education.

Concerning research question 4 , gamification is mostly studied to look at the impact on student engagement; thus also affecting student motivation and academic performance. When analyzing the studies in depth, most studies often mainly focused on the effect of the interaction/visualization with the user and a particular system. This might explain why engagement is a dominant dependent variable. Overall, it is questionable how researchers leave out important psychological and behavioral variables interacting with the gamification-human interaction. For instance, motivation is a key variable often mentioned by gamification researchers when designing a gamification experience [50], [51], [52], [53]. Nevertheless, it is striking that this variable is almost never explicitly measured in these studies. Moreover, when analyzing the data collection methods, it was found there is a lack of using validated psychometric instruments to measure the variables in most studies. Without them, the available results can be questioned due to their weak reliability. Very often, self-generated instruments are being used to measure the different variables. In line with the motivation example, one could use the intrinsic motivation inventory of Ryan [54], a well-known instrument to measure the different dimensions of motivation. Finally, in research question 5, unlike the findings in [10], we observe a positive tendency to require students spending more time in the gamification setting. At a more general level, sample size of most studies can be criticized in view of generalization and reliability of results. Working with a minimum of 100 students would give more statistical power to the studies and allow for better analysis approaches.

\section{LIMITATIONS}

Though we used a key scientific database to select research articles, the number of studies was fairly small and might not represent a complete overview of research available regarding gamification in Higher Education within a STEM context. Further research is required, building on additional literature databases (e.g., Scopus) to develop a more comprehensive picture of the field. Furthermore, since most studies did not build on an experimental design, involving control and experimental groups, a meta-analysis and a calculation of effect sizes could not be carried out.

\section{CONCLUSION AND FURTHER RESEARCH SUGGESTIONS}

This study presented a first overview of what research literature is available in terms of gamification within STEM fields in Higher Education. Although only 30 studies could be included in the analysis, it helped developing a first in-depth picture of recent developments in the field. The results showed: (1) Computer Science is the dominant STEM field being studied; (2) a combination of points, badges and leaderboards are mostly used; and (3) most studies see student engagement as the key dependent variable. These literature analysis results reflect clear gaps to be addressed in future research: controlled study of unique gamification elements to determine their individual effect on students; studies set up in other STEM areas; development of more complex models to study the impact of gamification by including mediating or moderating variables such as motivation, personality, and game preferences; and lastly, the design and adoption of high quality research instruments to develop valid and reliable research results.

\section{REFERENCES}

[1] Deterding, S., Dixon, D., Khaled, R., \& Nacke, L. (2011). From game design elements to gamefulness: defining gamification. In Proceedings of the 15th international academic MindTrek conference: Envisioning future media environments, pp. 9-15.

[2] Hamari, J. (2013). Transforming homo economicus into homo ludens: A field experiment on gamification in a utilitarian peer-to-peer trading service. Electronic commerce research and applications, 12(4), pp.236-245.

[3] Lister, C., West, J. H., Cannon, B., Sax, T., \& Brodegard, D. (2014). Just a fad? Gamification in health and fitness apps. JMIR serious games, 2(2). 
[4] Grant, S., \& Betts, B. (2013). Encouraging user behaviour with achievements: an empirical study. In Mining Software Repositories (MSR), 2013 10th IEEE Working Conference on (pp. 6568). IEEE.

[5] Denny, P. (2013). The effect of virtual achievements on student engagement. In Proceedings of the SIGCHI conference on human factors in computing systems (pp. 763-772). ACM. [6] Hanus M.D., Fox J. (2015). Assessing the effects of gamification in the classroom: A longitudinal study on intrinsic motivation, social comparison, satisfaction, effort, and academic performance, Computer \& Education, 80, 152-161.

[6] Dicheva, D., Dichev C., Agre G., \& Angelova G. (2015). Gamification in Education: A Systematic Mapping Study. Educational Technology \& Society, 18 (3), 75-88.

[7] Faiella, F., \& Ricciardi, M. (2015). Gamification and learning: a review of issues and research.Journal of e-Learning and Knowledge Society,11(3).

[8] Holdren, J. P., Marrett, C., \& Suresh, S. (2013). Federal Science, Technology, Engineering, and Mathematics (STEM) Education 5-Year Strategic Plan. National Science and Technology Council: Committee on STEM Education.

[9] Horizon 2020(n.d) The EU Framework for Research and Innovation. Retrieved from https://ec.europa.eu/programmes/horizon2020/en/h2020-section/science-education

[10] Hamari, J., Koivisto, J., \& Sarsa, H. (2014). Does gamification work?--a literature review of empirical studies on gamification. InSystem Sciences (HICSS), 2014 47th Hawaii International Conference on (pp. 3025-3034). IEEE.

[11] Seaborn, K., \& Fels, D. I. (2015). Gamification in theory and action: A survey. International Journal of Human-Computer Studies, 74, 14-31.

[12] Caponetto, I., Earp, J., \& Ott, M. (2014). Gamification and education: A literature review. InECGBL 2014: Eighth European Conference on Games Based Learning (pp. 50-57).

[13] Bodnar, C. A., Anastasio, D., Enszer, J. A., \& Burkey, D. D. (2016). Engineers at Play: Games as Teaching Tools for Undergraduate Engineering Students. Journal of Engineering Education.

[14] Pedreira, O., García, F., Brisaboa, N., \& Piattini, M. (2015). Gamification in software engineering-A systematic mapping. Information and Software Technology, 57, 157-168.

[15] Arksey, H., \& O'Malley, L. (2005). Scoping studies: towards a methodological framework.International journal of social research methodology, 8(1), 19-32.

[16] Johnson, L., Adams Becker, S., Cummins, M., Estrada, V., Freeman, A., and Ludgate, H. (2013). NMC Horizon Report: 2013 Higher Education Edition. Austin, Texas: The New Media Consortium.

[17] Johnson, L., Adams Becker, S., Estrada, V., Freeman, A. (2014). NMC Horizon Report: 2014 Higher Education Edition. Austin, Texas: The New Media Consortium.

[18] Johnson, L., Adams Becker, S., Estrada, V., and Freeman, A. (2015). NMC Horizon Report: 2015 Higher Education Edition. Austin, Texas: The New Media Consortium.

[19] de Sousa Monteiro, B., Gomes, A. S., \& Neto, F. M. M. (2016). Youubi: Open software for ubiquitous learning. Computers in Human Behavior, 55, 1145-1164.

[20] Cadavid, J. M., \& Gómez, L. F. M. (2015). Uso de un entorno virtual de aprendizaje ludificado como estrategia didáctica en un curso de pre-cálculo: Estudio de caso en la Universidad Nacional de Colombia. RISTI-Revista Ibérica de Sistemas e Tecnologias de Informação, (16), 1-16.

[21] Codish, D., \& Ravid, G. (2015). Detecting playfulness in educational gamification through behavior patterns. IBM Journal of Research and Development, 59(6), 6-1.

[22] Kim, S. (2015). Team Organization Method Using Salary Auction Game for Sustainable Motivation.Sustainability, 7(10), 14358-14370.

[23] Auvinen, T., Hakulinen, L., \& Malmi, L. (2015). Increasing Students' Awareness of Their Behavior in Online Learning Environments with Visualizations and Achievement Badges. Learning Technologies, IEEE Transactions on, 8(3), 261-273. 
[24] Hakulinen, L., Auvinen, T., \& Korhonen, A. (2015). The Effect of Achievement Badges on Students' Behavior: An Empirical Study in a University-Level Computer Science Course. iJET, 10(1), 18-29.

[25] Hakulinen, L., \& Auvinen, T. (2014, April). The effect of gamification on students with different achievement goal orientations. In Teaching and Learning in Computing and Engineering (LaTiCE), 2014 International Conference on (pp. 9-16). IEEE.

[26] Hakulinen, L., Auvinen, T., \& Korhonen, A. (2013). Empirical study on the effect of achievement badges in TRAKLA2 online learning environment. In Learning and Teaching in Computing and Engineering (LaTiCE), 2013 (pp. 47-54). IEEE.

[27] Haaranen, L., Ihantola, P., Hakulinen, L., \& Korhonen, A. (2014). How (not) to introduce badges to online exercises. InProceedings of the 45th ACM technical symposium on Computer science education (pp. 33-38). ACM.

[28] Sousa-Vieira, M. E., López-Ardao, J. C., Fernández-Veiga, M., Rodríguez-Pérez, M., \& LópezGarcía, C. (2015). Using Social Learning Methodologies in Higher Education. International Journal of Engineering Pedagogy, 5(2).

[29] Ibanez, M. B., Di-Serio, A., \& Delgado-Kloos, C. (2014). Gamification for engaging computer science students in learning activities: A case study. Learning Technologies, IEEE Transactions on, 7(3), 291-301.

[30] Mozelius, P., Collin, J., \& Olsson, M. (2015). Visualisation and gamification of e-learningattitudes among course participants. InICEL2015-10th International Conference on e-Learning: ICEL 2015 (p. 227). Academic Conferences and publishing limited.

[31] Sillaots, M. (2014). Achieving Flow Through Gamification in the Course of Computer Games. In European Conference on e-Learning (p. 470). Academic Conferences International Limited.

[32] Sillaots, M. (2014). Achieving flow through gamification: a study on re-designing research methods courses. In European Conference on Games Based Learning (Vol. 2, p. 538). Academic Conferences International Limited.

[33] Ejsing-Duun, S., \& Karoff, H. S. (2014). Gamification of a Higher Education Course: What's the fun in That?. In ECGBL2014-8th European Conference on Games Based Learning: ECGBL2014 (p. 92). Academic Conferences and Publishing International.

[34] Fabricatore, C., \& López, X. (2014). Using Gameplay Patterns to Gamify Learning Experiences. In ECGBL2014-8th European Conference on Games Based Learning: ECGBL2014 (p. 110). Academic Conferences and Publishing International.

[35] Akpolat, B. S., \& Slany, W. (2014). Enhancing software engineering student team engagement in a high-intensity extreme programming course using gamification. In Software Engineering Education and Training (CSEE\&T), 2014 IEEE 27th Conference on (pp. 149-153). IEEE.

[36] Pirker, J., Riffnaller-Schiefer, M., \& Gütl, C. (2014). Motivational active learning: Engaging university students in computer science education. In Proceedings of the 2014 conference on Innovation \& technology in computer science education (pp. 297-302). ACM.

[37] Caton, H., \& Greenhill, D. (2013). The effects of gamification on student attendance and team performance in a third-year undergraduate game production module. In European Conference on Games Based Learning (p. 88). Academic Conferences International Limited.

[38] Barata, G., Gama, S., Jorge, J., \& Goncalves, D. (2013). Engaging engineering students with gamification. In Games and Virtual Worlds for Serious Applications (VS-GAMES), 2013 5th International Conference on (pp. 1-8). IEEE.

[39] Barata, G., Gama, S., Jorge, J., \& Gonçalves, D. (2013). Improving participation and learning with gamification. In Proceedings of the First International Conference on gameful design, research, and applications (pp. 10-17). ACM.

[40] Burkey, D. D., Anastasio, M. D. D., \& Suresh, A. (2013). Improving student attitudes toward the capstone laboratory course using gamification. In Proceedings of 2013 Annual Conference and Exposition of the American Society for Engineering Education (pp. 3950-3968). Atlanta, GA: ASEE. 
[41] Long, J. N., \& Young, L. S. (2011). Multiplayer On-Line Role Playing Game Style Grading in a Project Based Software Engineering Technology Capstone Sequence. In American Society for Engineering Education. American Society for Engineering Education.

[42] Foster, J. A., Sheridan, P. K., Irish, R., \& Frost, G. S. (2012). Gamification as a strategy for promoting deeper investigation in a reverse engineering activity. In American Society for Engineering Education. American Society for Engineering Education.

[43] Berkling, K., \& Thomas, C. (2013). Gamification of a Software Engineering course and a detailed analysis of the factors that lead to its failure. In Interactive Collaborative Learning (ICL), 2013 International Conference on (pp. 525-530). IEEE.

[44] Prause, C. R., Nonnen, J., \& Vinkovits, M. (2012). A field experiment on gamification of code quality in agile development. In Psychology of Programming Interest Group Annual Conference (PPIG) (Vol. 2012).

[45] Singer, L., \& Schneider, K. (2012). It was a bit of a race: Gamification of version control. In Games and Software Engineering (GAS), 2012 2nd International Workshop on (pp. 5-8). IEEE.

[46] Leong, B., \& Luo, Y. (2011). Application of game mechanics to improve student engagement. InProceedings of International Conference on Teaching and Learning in Higher Education. doi(Vol. 10, No. 1.368, p. 1256).

[47] losup, A., \& Epema, D. (2014). An experience report on using gamification in technical higher education. In Proceedings of the 45th ACM technical symposium on Computer science education (pp. 27-32). ACM.

[48] O'Donovan, S., Gain, J., \& Marais, P. (2013). A case study in the gamification of a universitylevel games development course. In Proceedings of the South African Institute for Computer Scientists and Information Technologists Conference (pp. 242-251). ACM.

[49] Muilenburg, L. Y., \& Berge, Z. L. (Eds.). (2016).Digital Badges in Education: Trends, Issues, and Cases. Routledge.

[50] Zichermann, G., \& Cunningham, C. (2011).Gamification by design: Implementing game mechanics in web and mobile apps. "O'Reilly Media, Inc.".

[51] Chou, Y. K. (2015). Actionable Gamification: Beyond Points, Badges, and Leaderboards.

[52] Werbach, K., \& Hunter, D. (2012). For the win: How game thinking can revolutionize your business. Wharton Digital Press.

[53] Marczewski, A (2015). Even Ninja Monkeys like to play. Blurb Inc

[54] Ryan, R. M. (1982). Control and information in the intrapersonal sphere: An extension of cognitive evaluation theory. Journal of personality and social psychology, 43(3), 450. 